\title{
La actuación en el Centro Histórico de Gandía: urbanismo, comercio y colaboración pública-privada
}

Vicent Llorens Martí *

\section{Introducción}

En el año 1981, el Ajuntament de Gandía inicia la redacción del Plan Especial de Revitalización y Mejora del Medio Urbano de Gandía (PERYMMU).

El PERYMMU, que se aprobó definitivamente en el año 1984, fue el comienzo de una serie de iniciativas y actuaciones públicas y privadas que veinte años después han permitido situar a Gandía, en general, y al Centro Histórico, en particular, como una de las áreas comerciales más importantes de la Comunitat Valenciana.

La actuación en el Centro Histórico de Gandía, en todas sus vertientes, ha tenido como constante la relación de colaboración y participación establecida entre diversas administraciones públicas, entre ellas el Ajuntament de Gandía, la Diputación de Valencia, la Generalitat Valenciana, el Espacio Económico Europeo (compuesto por la Unión Europea y los países EFTA) e instituciones privadas, básicamente, la Federació de Comerciants de Centre Històric, los propietarios de inmuebles y los residentes en el Centre Històric y otros agentes económicos y sociales como la Cambra de Comerç de València y el Módulo de Comerç Interior del Institut Tirant lo Blanc de Gandía.

\section{La localización y marco general}

Gandía, con una población de 62.102 personas (según el Padrón de Habitantes del día 1 de enero de 2001), se encuentra situada en el litoral mediterráneo, a $65 \mathrm{~km}$. de la ciudad de València y a $116 \mathrm{~km}$. de Alacant, y ocupa un lugar destacado en la jerarquía de ciudades y áreas funcionales valencianas, inmediatamente detrás de las dos principales áreas metropolitanas regionales: València y Alacant-Elx.

Enclavada en el núcleo central del Mediterráneo, la ciudad se beneficia de esta estratégica situación, configurándose como un importante núcleo de comunicaciones.

La economía de la ciudad está basada en el sector de los servicios (el $70 \%$ de la población activa), aunque el sector industrial está en un proceso de crecimiento y diversificación.

Dentro del sector de los servicios destaca la actividad comercial, uno de los pilares básicos del desarrollo económico de la ciudad y que en los últimos años ha adquirido un papel estratégico: incrementando su área de mercado, aumentando la demanda extra-local y disminuyendo el gasto evadido. Valga como ejemplo que la población del área comercial de Gandía ascendía a 277.761 personas, con un mercado potencial local superior a los 22.000 millones de pesetas y con un mercado 
potencial del área superior a los 100.000 millones de pesetas, según el Anuario Económico de España 2001 de la Caixa.

Gandía se ha consolidado como el área comercial más importante de la Comunidad Valenciana, detrás de las tres capitales de provincia: Alacant, Castelló de la Plana y València, y por delante de ciudades que la superan en población como Elx y Torrent.

\section{El Centre Històric de Gandía: características y problemática}

El Centre Històric está formado por cuatro barrios con características propias:

a) La Vila, el barrio mediaval contenido en el primer recinto amurallado (s. XIII-XIV), con una superficie de $137.639 \mathrm{~m}^{2}$.

b) El Raval, el barrio medieval extramuros: la Morería i la Judería, coetáneo de la Vila y con un área de $83.680 \mathrm{~m}^{2}$.

c) La Vila Nova, el barrio renacentista ceñido en el segundo recinto amurallado (s. XVI), con una superficie de $78.691 \mathrm{~m}^{2}$.

d) L'Eixample, el ensanche urbano de finales del siglo XIX, en área de $111.040 \mathrm{~m}^{2}$.

En el comienzo de la década de los años ochenta el Centre Històric presentaba los siguientes rasgos:

a) Se conservaba buena parte de la trama urbana histórica aunque ésta había sufrido ya cambios y corría peligro de degradación y alteración definitiva.

b) El patrimonio edificado histórico, que mantenía un conjunto arquitectónico de gran interés, con diversas tipologías constructivas, propias de las características de cada uno de los barrios que conforman el Centre Històric, había experimentado significativas alteraciones desde finales de la década de los cincuenta, con la introducción de elementos arquitectónicos (previa demolición de edificios en la mayoría de las ocasiones) espurios a un centro histórico.

c) Existían edificios de gran interés histórico-artístico: la Seu Col.legiata y el Palau Ducal (monumentos nacionales); los restos del doble recinto amurallado; la antigua Universidad de los Jesuitas o el antiguo Hospital de Sant Marc, todos ellos en un deficiente estado de conservación.

d) Se mantenía la función residencial, aunque el Centre perdía población y se envejecía su pirámide demográfica.

e) El Centre Històric conservaba parte de sus funciones económicas tradicionales: comercio, servicios personales, profesionales, administración pública, etc., si bien perdía capacidad de competencia ante la aparición de nuevas centralidades urbanas, producto de la expansión de la ciudad, que acogían servicios públicos como el nuevo hospital comarcal y los nuevos juzgados, una vez habían abandonado éstos el Centre Històric.

\section{El Plan Especial de Revitalización y Mejora del Medio Urbano (PERYMMU)}

Coincidiendo con la elaboración de un nuevo Plan General de Ordenación Urbana de Gandía (el vigente en aquel momento databa de mediados de los sesenta), en el año 1981 y ante la problemática que se planteaba en el Centre Històric, el Ajuntament de Gandía inició la redacción del PERYMMU, que se aprobó definitivamente en el año 1984.

El objetivo genérico del PERYMMU (todavía vigente) es la protección del medio ambiente urbano y la conservación del conjunto urbano histórico-artístico.

Como objetivos específicos plantea los siguientes:

a) Evitar la pérdida de población del Centre Històric.

b) Conservar la trama urbana histórica y el patrimonio edificado.

c) Mejorar las condiciones ambientales del medio urbano.

Para la conservación de estos objetivos específicos, el PERYMMU contempla unos criterios de ordenación:

a) Conservación y revitalización de la estructura urbana histórica mediante la regulación de los elementos de la morfología urbana: trama viaria, volumetría, parcelación y edificaciones monumental y tipológica.

b) Conservación y revitalización de la estructura social: mantenimiento de la población residente, limitación de usos no residenciales, dotación de servicios e infraestructuras de interés social.

c) Conservación y revitalización de la estructura funcional: usos residenciales, usos comerciales y de servicios.

El PERIMMU fue aprobado con la unanimidad de todos los grupos políticos representados en el Ajuntament de Gandía. Sin embargo, podemos afirmar que en aquellos años esta iniciativa partió de una preocupación de carácter «ilustrado» ante la situación del Centre Històric más que una inquietud compartida por el conjunto de la ciudadanía y que no se concretaría hasta años más tarde. 


\section{El Plan de Reforma Urbana y el Programa de Actuación Comercial}

Entre los años 1987 y 1990 la Generalitat Valenciana inició, concretó y desarrolló el Programa de Ayuda a la Reforma y Mejora de Establecimientos Comerciales y el Programa sobre Actuaciones Protegibles en materia de Vivienda.

Estos programas de la Generalitat Valenciana a iniciativa de l'Ajuntament de Gandía (contando con el aval del PERYMMU en vigor) y de un pujante asociacionismo comercial en el ámbito del Centre Històric, se concretaron en el año 1989 en un convenio de colaboración entre la Generalitat Valenciana y el Ajuntament de Gandía para la ejecución del Programa de Renovación de la Ciudad Medieval de Gandía (PRU) que incluía el barrio de la Vila y el de la Vila Nova. En el año 1990 se inicia la primera fase de las actuaciones del PRU; en el año 1992 se aprueba la segunda fase y se declara de interés histórico-artístico y ambiental y en el año 1995 se amplía el ámbito de actuación del PRU, incluyendo acciones en El Raval y en L'Eixample.

A diferencia del PERYMMU, que tiene un carácter básicamente regulador, el PRU es un programa de actuaciones concretas y donde se habilitan instrumentos de financiación específicos.

Los objetivos del PRU son similares a los planteados en el PERYMMU:

a) Recuperar la trama urbana y el patrimonio edificado históricos.

b) Mejorar la función residencial del espacio urbano.

c) Favorecer el desarrollo de las actividades económicas tradicionales del Centre Històric.

Las actuaciones del PRU se han centrado, y se centran, en tres ámbitos concretos:

a) Rehabilitación y adecuación de fachadas, cubiertas, viviendas y bajos comerciales.

b) Renovación de la infraestructura viaria, redes de servicios, urbanización de espacios y equipamiento urbano.

c) Rehabilitación de edificios monumentales y singulares y dotación de equipamientos sociales y comunitarios.

Para viabilizar la ejecución de estas acciones, las administraciones públicas implicadas en las primeras fases del PRU (básicamente la Conselleria d'Obres Públiques, Urbanisme $i$ Transport, la Conselleria d'Indústria, Comerç $i$ Turisme, la Dipu- tació de València y el Ajuntament de Gandía) habilitaron medios de fomento de la inversión, por ejemplo:

- $60 \%$ de subvención máxima para adecuación de cubiertas y fachadas.

- Entre el $30 \%$ y el $60 \%$ de subvención para la rehabilitación de interiores para vivienda.

- $20 \%$ de subvención máxima para la remodelación de la fachada de bajos comerciales.

- $20 \%$ de subvención máxima para la transformación o nueva implantación de comercios.

- 37,5\% de subvención más un $20 \%$, si la calle o plaza tiene una implantación comercial mayoritaria, para la renovación de la infraestructura viaria (más conocido como «calles peatonales»), urbanización de espacios, renovación de las redes de servicios, dotación de mobiliario urbano.

- Alrededor del $60 \%$ de subvención máxima para la reha. bilitación de edificios monumentales y singulares para la instalación de equipamientos dotacionales.

- Exención en el pago de la tasa de licencia de obras.

- Exención en el pago de la tasa de ocupación de vía pública.

Asimismo, la Generalitat, la Diputació y el Ajuntament convinieron la instalación en Gandía de una oficina técnica y administrativa para tramitar con mayor agilidad las solicitudes de subvención.

Hasta el año 1995 (incluido) la ejecución del PRU supuso una inversión de 2.875.657.442 millones de pesetas, de las cuales la aportación privada fue del $54,9 \%$, la de la Generalitat del $24,6 \%$ y la del Ajuntament del $20,5 \%$.

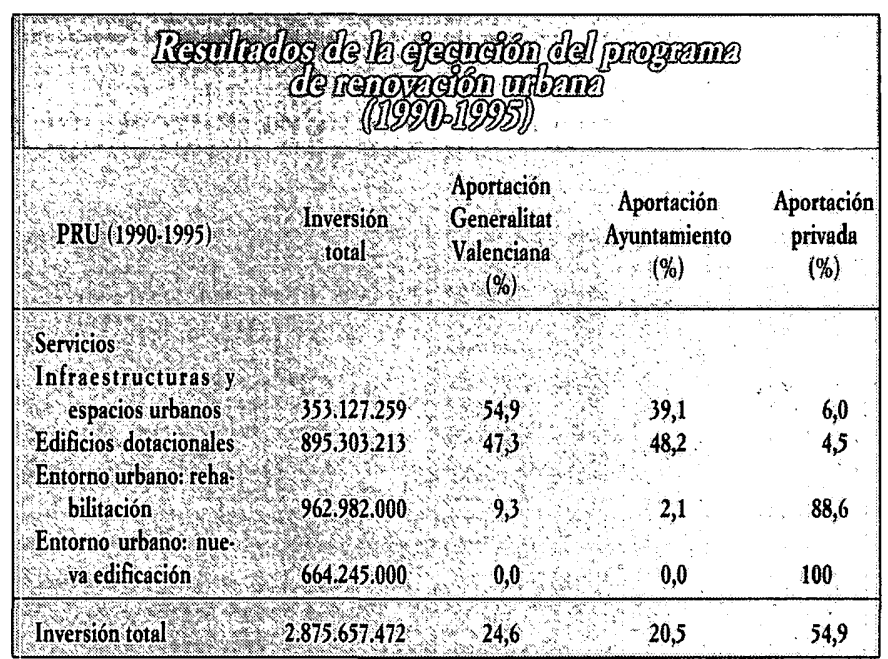

Fuente: Departamento de Urbanisme.

Desde el año 1996 hasta la actualidad disponemos de la información sobre la inversión realizada en infraestructuras 
y espacios urbanos y redes de servicio: 291.552 .499 millones de pesetas; en edificaciones dotaciones: 1.118.802.389 millones de pesetas, y en actuaciones que contribuyen a reforzar la centralidad del Centre Històric: 812.165. 735 millones de pesetas.

En paralelo al PRU se iniciaban también una serie de actuaciones tendentes a movilizar y dinamizar el sector comercial del Centre Històric.

En el año 1989 comenzó a trabajar el Centro de Desarrollo Comercial (CEDEC), convenido entre la Direcció General de Comerç y el Ajuntament, con los objetivos enunciados anteriormente.

Además, la Dirección General de Comerç financió la contratación de un gerente, especialista en comercio, por parte de la Federació de Comerciants de Gandía-Centre Històric, agrupación de asociaciones de comerciantes: Asociación de Comerciantes C/ Major y Adyacentes; Asociación de Comerciantes de Sant Francesc de Borja y Adyacentes y Asociación de Comerciantes C/ Sant Pasqual y Vicaris, con el objetivo, según sus Estatutos, «... de la representación y defensa de los intereses profesionales, económicos y sociales de las asociaciones que la integran, además de hacer cuanto sea posible para revitalizar comercialmente el Centre Histórico de la ciudad de Gandía».

El CEDEC junto con la Federació de Comerciants presentaron, en el año 1991, el llamado Programa de Actuación Comercial (PAC) con los siguientes objetivos:

a) El fortalecimiento y profesionalización del asociacionismo y la promoción de las acciones conjuntas del pequeño comercio.

b) La renovación de las estructuras comerciales.

c) La consecución del Centre Històric como un centro comercial de primer rango, determinando el mix comercial, profesionalizando y aumentando la competitividad del comerciante individual y promocionando y animando el Centre como cualquier centro comercial.

La ejecución conjunta del PAC entre la Federació de Comerciants y el CEDEC (a partir de 1996, la Agencia de Fomento de Iniciativas Comerciales, AFIC) desveló, por un lado, la necesidad de disponer de un programa de actuaciones conjuntas y de carácter integral para el desarrollo del proyecto del Centre Històric y que, al mismo tiempo, fuera coherente con los objetivos expresados en el PERYMMU y en el PRU y, por otro, se constató la eventualidad de incidir de manera especial en las actuaciones de carácter comercial.

La Federació de Comerciants y el Ajuntament de Gandía expusieron esta iniciativa a la Direcció General de Comerç, que acogió favorablemente la realización de un trabajo de estas características.

\section{El Proyecto del Centro Comercial Abierto}

En julio de 1993 se inicia la redacción del estudio de viabilidad técnico-económica y financiera sobre la «Evolución del Centre Històric de Gandía a Centro Comercial Abierto».

La financiación del trabajo corría a cargo de la Direcció General de Comerç y de la Direcció General d'Economia de la Generalitat Valenciana, de la Federació de Comerciants de Gandía-Centre Històric y del Ajuntament de Gandía.

La realización del estudio se contrataba, mediante concurso público, a una consultoría especializada, aunque la Federació de Comerciants y el Ajuntament constituyeron un equipo de trabajo para participar en la redacción del mismo. Este grupo estaba formado por técnicos de la Direcció General de Comerç, de la Cámara Oficial de Comercio de Valencia, por profesores del Módulo de Comercio Interior del Institut Tirant lo Blanc de Gandía, por comerciantes y técnicos de la Federació de Comerciants y por representantes técnicos y políticos del Ajuntament de Gandía.

El objetivo principal de este trabajo era elaborar un proyecto tendente a la creación de un Centro Comercial Abierto (CCA) en el entramado urbano del Centre Històric, tratando de trasladar las ventajas competitivas de un centro comercial cerrado a un espacio abierto. Así pues, se trataba de imprimir un cambio radical en la gestión de un espacio público, pasando de un concepto de gestión individualista y exento de elementos comunes a un sistema de gestión integrado, por una parte, por las empresas comerciales, los de servicios y por profesionales liberales en una unidad de gestión y, por otra, por la formalización de las relaciones de colaboración entre la mencionada unidad de gestión y el Ajuntament de Gandía.

Los objetivos instrumentales del proyecto del CCA eran:

a) Implantar una estructura de organización y gestión. Se optó por la fórmula jurídica de la cooperativa de servicios con dos funciones básicas:

- Vertebrar las actividades del CCA en un conjunto de áreas funcionales.

- Celebrar acuerdos o convenios de colaboración con diferentes organizaciones tendentes al correcto desarrollo del proyecto del CCA.

Para la consecución de estos fines se propone el desarrollo de dos programas de actuación:

- Administración y gestión de la sociedad.

- Comunicación interna. 
b) Mejora y promoción del conjunto comercial del Centre Històric:

Primero, a través de la mejora de la competitividad de los establecimientos, para lo cual se desarrollan tres programas concretos de actuación:

- de Formación;

- de Innovación;

- de Servicio a las empresas.

Segundo, mejora del poder de actuación comercial del Centre Històric a través de la ejecución de programas sobre:

- Infraestructuras urbanas, tráfico y accesibilidad.

- Entorno interior del Centre: normativa reguladora de las actividades que se dan en la zona; seguridad; limpieza; mantenimiento del equipamiento urbano, etc.

- Delimitación e identificación de la zona: implantación de la imagen comercial del CCA.

- Mix comercial del CCA.

- Comunicación externa: publicidad y promoción; animación comercial; organización de actos culturales, deportivos, etc.; relaciones públicas y presencia en foros sobre comercios, centros históricos, etc.

- Servicios a los consumidores.

- Investigación y desarrollo.

Podemos concluir que la realización del estudio de viabilidad sobre la «Evolución del Centre Històric de Gandía a Centro Comercial Abierto», que concluyó a finales del año 1994, ha comportado la concertación de las actuaciones que se debían materializar en el ámbito de la zona. Además, esta concertación se ha producido a dos niveles: en primer lugar, entre las propios comerciantes $y$, en segundo lugar, entre los comerciantes y el Ajuntament.

\section{7. «Gandía Comercial, Centre Històric, Cooperativa Valenciana» y la colaboración con el Ajuntament de Gandía}

La materialización formal del convenio sobre las acciones a desarrollar en el ámbito del Centre Històric se produce como apuntábamos, a dos niveles:

- La Federació de Comerciants constituye, en julio de 1995, una cooperativa de servicios «Gandía Comercial, Centre
Històric, Coop. V.» como instrumento de desarrollo del proyecto del CCA.

- En mayo de 1996 se suscribe entre «Gandía Comercial, Centre Històric, Coop. V.» y el Ajuntament de Gandía el primer convenio de colaboración para el desarrollo del proyecto del CCA. Este convenio de colaboración se ha renovado en dos ocasiones: en el año 1997 y en el año 2001.

Una de las conclusiones del proyecto del CCA ha sido la constitución de una cooperativa de servicios como la estructura más adecuada para gestionar la participación de los comerciantes en el CCA. Las razones de la elección de esta fórmula jurídica son múltiples: pueden ser socios los que por razón de su actividad cubren el objetivo social y las entidades públicas; cada socio cuenta con un voto, con independencia de su participación en el capital social; permite una aportación diferenciada entre los establecimientos de forma justa y equilibrada; posibilita la suscripción de convenios y la recepción de subvenciones; favorece un desembolso inicial mínimo; goza de una fiscalidad ventajosa; puede beneficiarse de programas de promoción y fomento del cooperativismo de la Generalitat Valenciana; es una fórmula jurídica conocida y valorada positivamente por el empresario de la zona, etc.

$\mathrm{La}$ Cooperativa ofrece una serie de servicios tanto para las empresas asociadas como para los consumidores. Por ejemplo: información, asesoramiento y tramitación de ayudas directas e indirectas; bolsa de trabajo; asesoramiento técnico en cuestiones comerciales; servicios de negociación ventajosa con entidades financieras, aseguradoras, etc.; reparto a domicilio; programación de formación continua para empresarios y trabajadores; campañas de promoción y animación comercial a lo largo del año en Gandía y su área de mercado; taller de composturas; parque de ocio infantil y tren turístico; control y seguimiento del mix comercial y captación de empresas comerciales y de ocio para instalarse en el Centre Històric; resolución de conflictos entre comerciantes y consumidores mediante Sistemas de Arbitraje y Conciliación (actuando habitualmente la Cooperativa como representante del comercio reclamado); aparcamiento subterráneo gratuito mediante tiques ofrecidos por la Cooperativa; colaboración en la vigilancia del Centre Històric con la Policía Nacional y Local; ayuda al Ajuntament en el control de los horarios y de las zonas de carga y descarga; comunicación de cualquier incidencia acaecida en el ámbito del Centre Històric, etc.

«Gandía Comercial, Centre Històric Coop.V.» se constituyó en el año 1995 con unos sesenta asociados y en la actualidad cuenta con más de doscientos, arrojando el último balance del ejercicio del año 2000 un resultado positivo.

Tras la constitución de la Cooperativa la relación con el Ajuntament se formaliza mediante un convenio de colaboración suscrito el día 17 de mayo de 1996. El objetivo del Convenio 
era «establecer el marco de colaboración necesario para la mejora del comercio de centro histórico de Gandía, que esté integrado dentro del ámbito de actuación de la cooperativa "Gandía Comercial, Centre Històric, Coop. V.", propiciando de forma preferente el adecuado y progresivo desarrollo del Centro Comercial Abierto...».

El Convenio establece una serie de compromisos tanto para el Ajuntament como para la Cooperativa. El Ajuntament se compromete a ejecutar todas aquellas actuaciones de tipo arquitectónico, urbanístico y de gestión de la accesibilidad que están recogidas en el proyecto del CCA: aprovechamiento, adecuación y mejora de la infraestructura urbanística existente; tráfico rodado y aparcamientos; calles de uso preferentemente peatonal; actividades y usos permitidos; mejora de los accesos al CCA; mantenimiento y limpieza de los elementos urbanos y comunes del CCA; seguridad y vigilancia de la zona; condiciones de uso e implantación del nombre comercial o marca del CCA (propiedad del Ajuntament), etc. Y además a aportar durante tres años (1996, 1997 y 1998) la cantidad de 44.800.000 pesetas a la Cooperativa en concepto de ayudas, subvenciones y servicios para la puesta en marcha y lanzamiento del proyecto empresarial-comercial.

La Cooperativa, por su parte, se obliga a desarrollar la gestión de las distintas áreas funcionales propias de un centro comercial y contenidas en el proyecto del CCA: administración eficaz del CCA; desarrollo de servicios de carácter técnico para los asociados: asesoramiento de mercado, negociación ventajosa de servicios, creación de nuevas empresas, consecución de condiciones de compra favorables para los comercios, etc., programas de formación técnica y profesional de los socios; campañas de animación y promoción comercial; información y publicidad; implantación de sistemas de gestión ambiental del comercio; utilización del valenciano en la promoción y publicidad del CCA, en otras acciones, muchas de ellas ya relacionadas.

Asimismo, el Convenio establece una comisión de seguimiento encargada de vigilar «el adecuado cumplimiento de los fines del proyecto de creación e implantación de un Centro Comercial Abierto» e integrada por nueve miembros: dos representantes de la Cooperativa; un representante de la Direcció General de Comerç de la Generalitat Valenciana; un representante de la Cambra Oficial de Comerç, Indústria i Navegació de València; un representant del Mòdul de Comerç Interior del Institut Tirant lo Blanc; dos representantes de las centrales sindicales más representativas y dos representantes del Ajuntament.

Las funciones de esta Comisión son, entre otras: interpretar el Convenio y los anexos que lo desarrollan; resolver posibles controversias; informes de cuantos temas puedan ser de interés para el desarrollo del proyecto y la aplicación del Convenio; acordar y proponer a las partes firmantes posibles actuaciones que deban incluirse en sucesivos anexos, etc.

El Convenio de colaboración suscrito en el año 1996 fue modificado por un nuevo Convenio firmado el 17 de noviembre de 1997, «en aras de un desarrollo más eficaz del mismo». En concreto se suprime la aportación del Ajuntament de Gandía en especie (exoneración del canon de ocupación de vía pública por actividades de animación y promoción comercial; refuerzo del servicio de policía en el Centre Històric; cesión de locales para actividades de formación, etc.) por existir discrepancias insalvables sobre la valoración económica de la misma y se sustituye por una aportación económica del total de 27.490.000 pesetas hasta el año 1999, incluido (a esta cantidad había que añadir la aportación ya realizada al año 1996 de 14.500 .000 pesetas). Hasta el año 2000 el Ajuntament de Gandía ha aportado una cantidad superior a los 44 millones de pesetas.

Además, se modifica la comisión de seguimiento, limitando su composición a tres representantes de la Cooperativa y a tres del Ajuntament, contemplando la posibilidad de participar, previa invitación, bien de la Cooperativa, bien del Ajuntament, representantes de la Direcció General de Comerç, de la Cambra Oficial de Comerç, del Mòdul de Comerç Interior del Institut Tirant lo Blanc o de las centrales sindicales más representativas. Las razones para cambiar su composición cabe hallarlas en cuestiones de operatividad y agilidad. Por lo demás, se mantienen sus objetivos y sus funciones.

La valoración que podemos realizar del trabajo realizado por la Cooperativa, así como de la gestión del convenio de colaboración es realmente positiva, para muestra un botón: en el año 1998 «Gandía Comercial, Centre Històric, Coop. V.» recibía el Premio Nacional de Comercio Interior al Pequeño Comercio de la Secretaría de Estado de Comercio y Turismo y de la Pequeña y Mediana Empresa del Ministerio de Economía y Hacienda (BOE, núm. 313, 31 de diciembre de 1998).

La vigencia del Convenio de colaboración firmado el año 1997 finalizaba en diciembre de 1999, existiendo la posibilidad de extinguirlo si alguna de las partes así lo solicitaba durante los seis primeros meses del año 2000. Sin embargo, ninguna de las dos partes solicitó la extinción del mismo, como prueba de la predisposición a continuar una estrecha relación de colaboración, si bien los términos de la misma debían cambiar necesariamente: el Centre Històric se ha consolidado como un área estratégica de comercio evolucionado, como una empresa comercial moderna, concienciada de la conveniencia de la colaboración intrasectorial, con las administraciones públicas y con los demás agentes económicos y sociales de la zona, receptiva a la necesidad de la racionalización y de la innovación en la gestión y con una decidida vocación de servicio.

Así pues, con nuevos elementos y nuevas situaciones a considerar, en el año 2000 se ha llevado a cabo la negociación 
de un nuevo convenio de colaboración firmado el pasado 12 de febrero. Se ha considerado, por una parte, la consolidación del área comercial del Centre Històric y del proyecto empresariäl de «Gandía Comercial, Centre Històric, Coop. V.». Y por otra parte, la ejecución del PRU que, a través de sus diversas actuaciones de bonificación urbanística y arquitectónica, ha permitido el mantenimiento de la población del Centre Històric, el rejuvenecimiento de su pirámide demográfica, la atracción permanente de un importante número de usuarios a los equipamientos dotacionales habilitados en el ámbito de intervención, la mejora de las condiciones para el desarrollo de las actividades comerciales, con un aumento significativo del número de establecimientos, y el incremento de las actividades de servicios a empresas y personas así como los usos turísticos de la zona.

En este contexto de viabilidad económica y social del proyecto del Centre Històric resultaba difícil mantener una subvención económica del Ajuntament a la Cooperativa, por lo que ambas partes han acordado, a tenor de la mejora experimentada en la zona (después de más de diez años de actuaciones de carácter público) para el desarrollo de las funciones residenciales, comerciales y de servicios, la modificación de la Ordenanza Fiscal del Impuesto de Actividades Económicas (IAE) en lo que respecta a la Categoría y al Índice de Situación de aquellas calles y plazas que, objetivamente, se han beneficiado de las actuaciones realizadas. Se trata, al fin y al cabo, de adecuar su situación fiscal a la situación real después de las mejoras experimentadas, considerando las inversiones realizadas y que redundan en beneficio de las actividades económicas allí implantadas.

El incremento de la recaudación por la modificación del IAE ha sido de unos 27 millones de pesetas, que es la cantidad que el Ajuntament se ha comprometido a aportar anualmente a la Federació de Comerciants del Centre Històric de Gandía hasta el año 2005, incluido, según el convenio de colaboración vigente. Por lo demás, éste se mantiene prácticamente igual que el convenio de 1997 en cuanto a las acciones a desarrollar por la Federació de Comerciants del Centre Històric de Gandía, y por el Ajuntament, la comisión de seguimiento, etc.

Lo verdaderamente significativo de esta nueva relación ha sido el compromiso por parte del Ajuntament de aumentar, con la unanimidad de todos los grupos políticos, la presión impositiva en el ámbito del Centre Històric, a pesar del coste público de este tipo de medidas y la madurez de la mayoría de las empresas comerciales y de servicios ubicadas en el Centre Històric que han acogido de manera positiva este aumento de la presión fiscal. Con esta medida, además, se consigue la con- tribución económica de todas las empresas radicadas en el Centre Històric, y objetivamente beneficiarse del desarrollo de este proyecto, evitando así actitudes y comportamientos insolidarios.

\section{A modo de conclusión}

El proyecto del Centre Històric-Centre Comercial Obert de Gandía reúne otras cuestiones, en estos momentos incipientes y en desarrollo, en la concreción de las cuales también ha tenido un papel decisivo la colaboración entre la Federació de Comerciants y el Ajuntament:

1. El plan de tráfico del Centre Històric; la movilidad y las condiciones de accesibilidad al mismo; la gestión de las actividades y de las zonas de carga y descarga, etc., materializados en la Ordenanza Reguladora del Tránsito Urbano a la ciudad de Gandía (en vigor desde el 8 de julio de 2000), y el Plan de Ordenación del Tráfico Urbano que consagran el Centre Històric como una zona de uso preferentemente peatonal, permitiendo el acceso motorizado a residentes, comerciantes y repartidores en unas condiciones y en un horario determinados mediante la gestión de una serie de pivotes hidráulicos que impiden o permiten, según sea el caso, el paso de vehículos.

2. ${ }^{\circ}$ Evitar la especulación sobre solares y edificios del Centre Històric mediante la aplicación en Gandía del llamado Registro Municipal de Solares, contenido en la Ley Valenciana Reguladora de la Actividad Urbanística, y que obliga a los propietarios de solares o de edificios en estado de ruina en el ámbito del Centre Històric, a presentar proyectos de construcción en unos plazos determinados y, en caso de incumplimiento, permite la actuación subsidiaria por parte de la Administración.

Podemos afirmar que esta acción que se viene aplicando en Gandía en los últimos años está dando ya resultados positivos, evitando la permanencia de solares y edificios como actividad especulativa y agilizando la presentación y realización de proyectos de edificación.

No vamos a repetir los aspectos positivos del proyecto del Centre Històric-Centre Comercial Obert en cualquiera de los ámbitos de su intervención; tan sólo destacar que sin la colaboración entre la Federació de Comerciants del Centre Històric y el Ajuntament de Gandía este conjunto de actuaciones no hubiera tenido lugar y manifestar, como inquietud, y por lo que pueda competer a todas y cada una de las partes implicadas, la escasa participación de carácter organizado de los vecinos en este proyecto, que esperamos se enmiende con la recién constituida Associació de Veïns del Centre Històric de Gandía. 
" Geógrafo y Técnico Superior de la Promoción Económica del Ayuntamiento de Gandía. 